\title{
La Teoría de la Educación en la actividad académica española. Análisis de un indicador ${ }^{1}$
}

\author{
JOAQUÍN GARCÍA CARRASCO \\ ANGEL GARCÍA DEL DUJO \\ ANGELA BARRÓN RUIZ \\ MARGARITA GONZÁlEZ SÁNCHEZ \\ Universidad de Salamanca
}

\begin{abstract}
SUMMARY.-Analysis of the denomination of the courses and programs offered at a large number of Spanish universities enabled the authors to identify the different types of internal configuration that the field of Theory of Education acquires at the different institutions, together with the multiple thematic focal points of interest for research and teaching at each university. Some of the problems inherent to the programming of course content are also dealt with.
\end{abstract}

El título del trabajo es muy explícito. Pretendemos reflexionar sobre la actividad académica de enseñar teoría de la educación. Esta actividad tiene indicadores más propios y relevantes que el que se analiza aquí: textos escolares, libros publicados por los profesores, artículos en revistas... etc. Alguien debiera llevar a cabo lo que en otros sitios se denomina «revisión» y revisar con esmero nuestro entorno; mucha producción valiosa se menosprecia porque no se aporta a la lectura la atención y consideración necesaria.

No sugerimos la revisión por prejuzgar nada negativo, con los años vamos siendo cada vez más conscientes y respetuosos con el valor de lo posible; antes al contrario, creemos que la no consideración atenta de la producción de los compañeros de gremio, la falta de cooperación en la producción científica, la inexistencia de equipos sólidos de trabajo en las instituciones, la atribución de existencia a innumerables líneas de investigación (en algunos lugares hay «bastantes» indicadas y profesadas por un sólo investigador)... etc., puede ser todo ello indicadores de «escasez vital» y de un aislamiento intelectual presuntuoso que se contenta únicamente con la publicidad y la crítica ante el espejo.

1. Una primera versión de este trabajo fue presentada y comentada en el X Seminario Interuniversitario de Teoría de la Educación de Oviedo (diciembre de 1991). 
Tampoco quieren estas reflexiones entrar en la discusión soterrada -más historia de escalera que debate serio- sobre si la «teoría de la educación ha tocado techo o fondo». Con ello se quiere indicar que el crecimiento cuantitativo de profesionales del gremio aumentó con puestos de trabajo (no se olvide que el puesto es lugar, sitio, nada más) cuya denominación tenía más que ver con Teoría que con otras cosas. Ahora, alguno podría pretender que la dirección del pensamiento pedagógico, para ir más deprisa y ganar tiempo, en vez de orientarla mediante el debate serio hay que maniobrarla de forma más drástica y contundente: aislar grupos y promover desarrollo selectivo, autosegregación de agrupamientos profesionales, magnificar los impedimentos y negar la participación si no se encuentra controlado el liderazgo de las iniciativas... A nadie, sin embargo, se le puede ocultar que «teorizar» es quizás el rasgo y característica más común y advertible del quehacer universitario en relación a cuestiones pedagógicas. Lo que más hacemos los pedagogos académicos, además de satisfacer nuestras necesidades humanas y culturales, es hacer "teoría de la educación»; también aquí, como decía Ortega, cabe afirmar que hacer teoría de la educación (él decía «filosofar») es algo inevitable. Por el contrario, si teorizar es ineludible, o andamos despiertos o se nos escapa, también a todos, la oportunidad más próxima, inmediata, de aplicación y ensayo de la racionalidad y las técnicas pedagógicas: el valor «práctico» del mismo quehacer de enseñar pedagogía, incluso Teoría de la Educación. No son nuestras Facultades de Educación dechados de praxis pedagógica, ni ojos de huracán de innovaciones didácticas, ni están diseñadas para incentivar la actividad en esa dirección. Al menos le faltan dos elementos que consideramos fundamentales: laboratorios y centros de observación y aplicación; mientras se anda entre quimeras de grupos se van perdiendo oportunidades de edificación; hoy los edificios científicos no se pueden hacer más que en equipo.

Lo que pretenden estas líneas es demostrar que la comunicación científico-institucional es insuficiente, que puede mantenerse cierta deriva pedagógica de los planes de estudio, que acecha la falta de consenso en la terminología y la pequeña y equivocada presunción de creer estar empeñado en el pensamiento creativo por el mero hecho de dedicarse a buscar nombres nuevos a lo que cada uno haga. Las consecuencias más graves de este estado de cosas pueden ser las siguientes: (i) planes de estudio empobrecidos porque no siempre se plantean sobre razón sino sobre ocupación, no se mira tanto en algunos casos si se cubre la información sobre un campo necesario, sino si se ha dejado bien amojonada la parcela de la influencia; (ii) escasa presencia de material didáctico para uso de los estudiantes -manuales-; (iii) excesiva valoración de lo que se da en llamar «mi propuesta», «mi aportación»... «mi...», que retiene mucha actividad en cuestiones meramente generales y al mismo tiempo pretendida y buscadamente diferenciables, con lo que difícilmente se agotan corrientes -mueren antes de haber nacido-, y se camuflan las cuestiones reales en cuestiones de palabras, denominaciones y definiciones; (iv) poco crédito interno, porque a la evidencia del valor de lo que cada uno aporte se le inyecta en ocasiones algún que otro prejuicio o se le anticipa la opinión de algún vecino.

\section{CiRCUNSTANCIAS QUE JUSTIFICAN ESTE TRABAJO}

La intención de este trabajo se construye a partir de varias circunstancias.

(i) En los Centros de enseñanza superior, en España, se están planteando nuevos planes de estudio y nuevas titulaciones. 
(ii) La necesidad de la comparación y el contraste entre las diferentes propuestas que en cada Universidad se ofrecen, dado que ésto constituye, al menos implícitamente, un dato objetivo para la identificación del conjunto de problemas que los especialistas españoles consideran ámbito disciplinar de su competencia y necesidad informativa en la formación de quienes hayan de trabajar profesionalmente en los diferentes campos educacionales.

(iii) Un requisito para la configuración de grupos de trabajo donde se intercambien experiencias, se definan líneas de investigación que permitan la cooperación y el diálogo inteligible.

\section{EL TÉRMINO TEORÍA DE LA EDUCACIÓN EN EL USO ESPAÑOL}

Frente a esta obviedad de la intención juegan en contra algunas ambigüedades y polisemias.

(i) El término Teoría de la Educación se incluye como término de identificación de lo que en la organización española se denomina «Área de conocimiento».

(ii) El término Teoría de la Educación se emplea para identificar una «disciplina» particular dentro de «algunos» planes de estudio.

(iii) El término Teoría de la Educación también se emplea para identificar un ámbito proposicional, una construcción conceptual, que pretende decidir sobre la naturaleza epistemológica del discurso pedagógico en su totalidad y sobre la naturaleza del sistema de procesos más envolventes que constituyen los fenómenos educacionales tomados en su conjunto. Pretende tener un significado análogo epistemológicamente a cuanto se emplea como teoría general o teorías particulares en otros ámbitos del conocimiento, independientemente de la sintaxis o forma que adquiere en el ámbito de las Ciencias Humanas y en particular de la educación.

Qué pueda ser una T.E. es una preocupación constante de muchos especialistas, filósofos, pedagogos, sociólogos, antropólogos... Qué deba ser es una cuestión que pretende resolverse presionando en dos direcciones contradictorias, una centrífuga-expansiva y otra centrípeta-reductiva. En la primera intervienen preocupaciones de exposionan la incidencia de los profesionales en los espacios académicos, y en la segunda-desde otros presupuestos- intervienen criterios más práxicos o pragmáticos que reducen esta necesidad a un puñado de problemas generales o relativiza la adjudicación a especialistas de los mismos aplicando el criterio de que de teoría educativa tiene necesidad, y tiene que ocuparse, todo el mundo; con lo que se difumina la posibilidad de aplicación del concepto de área, de disciplina y de clara identidad del especialista. Por más que no se pueden poner barreras al viento ni tope ni límite a la curiosidad intelectual de nadie.

\section{DE LA OBSERVACIÓN SE DEDUCEN TRES FORMAS O CATEGORÍAS DE «TEORÍA»}

Lo que ciertamente está claro es que nos encontramos, de hecho, con tres modos de teorizar en el campo de la educación:

(i) Teoría-I. Se elaboran constructos intelectuales acerca del sistema de referentes del concepto de educación, asumido como totalidad, y, para hacerlo, se recogen materia- 
les construidos ad hoc, y materiales, -principalmente filosóficos, antropológicos, psicológicos, muy generales- a los que se le aplican las modulaciones necesarias para ser transferidos a este campo. Ejemplos podrían ser: Moore, Novak... y muchos trabajos de autores españoles acerca de la causalidad en la educación, la finalidad... etc. La T.E. intenta reproducir y semicalcar, respecto a la educación, la reflexión epistemológica que se aplica a otras ciencias cuando se reflexiona y escribe sobre Teoría de la Biología, Teoría de la Sociología... En general, se pretende tipificar la forma de pensamiento en ese campo del conocimiento, la tipología del método dominante en el progreso de conocimiento, el carácter de sus generalizaciones o leyes, en resumen, el valor de verdad de sus proposiciones. Estos materiales se han producido en todos los aledaños de la Pedagogía -Antropología, Sociología, Psicología...- y, también, ha sido objeto de muchos trabajos de pedagogos: es lo que se suele agrupar como trabajos de epistemología de las Ciencias de la Educación.

(ii) Teoría-II. Se elaboran los constructos educacionales recogiendo teorías sectoriales de muchos campos científicos - principalmente psicología- en los que se indagaba sobre la naturaleza de las estructuras que soportan el comportamiento y sus mecanismos de funcionamiento, y se inducen aplicaciones a contextos de acción humana concreta planteando los criterios normativos por los que debe dirigirse la influencia o el diseño de acción, en vistas a conseguir rendimiento óptimo del curso de acción en cada situación. Aunque algunos demuestren que este modo de hacer ha caracterizado alguna corriente concreta de pensamiento (v.g. conductismo), implícitamente, al menos, se puede rastrear este modo de hacer desde que tenemos noticias escritas sobre asuntos educacionales, hasta el modo de proceder de los mismos detractores del planteamiento. De hecho, incluso hoy, se encuentra entre personas cuya orientación conductista es injustificada. Esta voluntad teórica de iluminar la acción pedagógica ha tenido tres partituras, cada una de las cuales con diferente distanciamiento real del quehacer en el que se encuentran prendidos quienes no tienen otro remedio que actuar mientras los otros se encuentran reflexionando: (i) «Comprender» los fenómenos o procesos o hechos o situaciones educacionales; comprender ha significado cosas muy diferentes en épocas distintas y en escuelas o sistemas filosóficos diferentes, $\mathrm{y}$ hasta tiene sentido no superponible en las ciencias naturales y en las humanidades; digamos que funcionalmente se orienta a "explicar" lo que pasa. (ii) Contrastar, criticar la realidad de los hechos y estados de cosas con el acontecimiento posible o proyectado, y tomando como herramienta el conocimiento disponible, ir corrigiendo el curso de acción o alimentándolo con una secuencia de decisiones, la cual cumple un cometido de intervención y produce un beneficio de conocimiento, de aprendizaje en la acción. Si a ello se añade la complejidad de factores -estructurales, personales, circunstanciales, históricos, culturales...- que intervienen en el derrotero de cada cual, se comprende la variedad de discursos coherentes que pueden darse. Cualquiera de ellos debe asumir tres cosas, al menos: que el sistema comportamental humano es modulable, que la modulación puede ser interferida mediante la intervención de agentes personales o sociales, y que esa interferencia no es ni determinante ni meramente aleatoria.

(iii) Teoría-III. No se puede dejar de lado que la T. E. también se practica, con incidencia mucho mayor en las situaciones educativas que las dos anteriores, partiendo de las teorias personales de los propios educadores, las cuales se elaboran (i) mediante ra- 
cionalizaciones de experiencias vividas y acumuladas, (ii) mediante la transferencia del propio esquema de interpretación de la comunicación y la relación interpersonal a las situaciones educativas, (iii) mediante la aplicación de los sistemas de conceptos aprendidos, (iv) mediante la inferencia de criterios que se construyen y elaboran a partir de la inevitable reflexión sobre la práctica educativa vivida. Esta última, sólo por un apriorismo ingenuo, podemos estimar que siempre se ha de dirigir hacia constructos valiosos y teóricamente aprovechables; la reflexión sobre la práctica es, también, la que fija la rutina, reclausura los mecanismos de resistencia a la innovación, argumenta el rechazo de las propuestas innovadoras.

\section{LUGAR DE LOS PROGRAMAS TEÓRICOS SOBRE LA EDUCACIÓN EN ESTE CONTEXTO}

El presente trabajo no tiene otra intención que la de reflexionar, en el contexto de los presupuestos anteriores, partiendo de la lectura de los programas de disciplinas, cuyo diseño y ejecución fue entendido como ejercicio pedagógico de la actividad de construir, y formar con ella, una T.E.

Los materiales utilizados proceden de las Universidades siguientes y llevan el rótulo o título que se indica. No indicamos el autor, porque su identificación no añade nada relevante a lo que pretendemos llevar a cabo.

- Universidad de Oviedo (O).

- Universidad de Granada (G).

- Universidad Autónoma de Barcelona (AB).

- Universidad de Madrid (M).

- Universidad de Sevilla (S).

- Universidad de Barcelona (B).

- Universidad de Santiago de Compostela (SC).

- Universidad de Salamanca (SA).

- Universidad de Deusto (D).

- Universidad de Málaga (MA).

- Universidad Nacional de Educación a Distancia (UD).

\section{Las denominaciones disciplinares}

Las denominaciones disciplinares de los documentos estudiados son las que enumenamos a continuación. Hemos descartado las denominaciones de los cursos de doctorado, porque introducen muchas más denominaciones, y harían mucho más compleja la presentación. También excluimos todos los programas de Historia de la Pedagogía, Educación Comparada, Organización Escolar... etc. No por entrar y tomar partido acerca de la cuestión de dónde deben situarse para su gestión académica, ni dónde adscribirse administrativamente los profesionales, sino porque nuestra preocupación es racional y disciplinar. Y es obvio que esas otras cosas se confeccionan a partir de otros materiales. Lo que no nos impide decir que identificar T.E. como indicativo y lema de un Area académica de las aludidas en las leyes españolas, con T.E. en tanto que denominación de una disciplina académica, además de un disparate nos parece malintencionado. Y aplicado a 
todo y todos nos lleva a tantas áreas razonablemente como disciplinas académicas: absurdo. La lista que sigue se refiere más a adjudicaciones a Departamentos, ocupaciones de gentes calibradas para pertenecer al Area de T.E.: lo que hacen de hecho.

(1) Pedagogía General (O.1) (M.2) (S.1) (SC.3) (D.2) (MA.2).

(2) Sociología de la Educación (O.2) (AB.9) (S.5) (B.9) (SC.5) (SA.4).

(3) Pedagogía Sistemática (G.1).

(4) Introducción a las Ciencias de la Educación. ... a la Pedagogía (G.2) (AB.4) (B.3) (MA.1).

(5) Filosofía de la Educación (G.3) (M.2) (B.5) (D.3) (MA.3).

(6) Política y legislación educativa en España,... y Administración educativa... Planificación (G.4) (AB.7) (S.3) (SA.2).

(7) Supuestos de la educación (G.5).

(8) Biología de la Educación (AB.1).

(9) Condicionamientos biológicos del niño deficiente (AB.2).

(10) Educación permanente y de adultos (AB.3) (S.4).

(11) Pedagogía Social (AB.6) (M.4) (B.9b) (SC.5) (D.1) (MA.4) (UD.2).

(12) Sistemas de Educación no formal (AB.8).

(13) Teorías de la Educación. Teoría de la Educación (AB.10) (S.2) (B.1) (SC.4) (UD.1) (SA.1).

(14) Fundamentos de Metodología (M.1).

(15) Etica y Política de la educación (M.3).

(16) Teoría del aprendizaje (B.2).

(17) Pedagogía Cibernética (B.4).

(18) Educación Moral (B.6).

(19) Pedagogía ambiental. Educación ambiental (B.7) (SC.5).

(20) Investigación observacional en la Acción educativa (B.8).

(21) Teoría de la Comunicación y Pedagogía (B.4b).

(22) Antropología Pedagógica (B.5b).

(23) Pedagogía Internacional (B.5c).

(24) Información, educación y ordenadores (B.6b).

(25) Animación socio-cultural y Pedagogía del ocio (B.8b).

(26) Pedagogía contemporánea (B.10).

(27) Pedagogía gerontológica (B.11).

(28) Educación y Motivación (SC.1).

(29) Reformas e innovación en el Sistema Educativo (SC.2) (SA.3).

(30) Educación y desenvolvimiento personal (SC.5).

(31) Técnicas de investigación socioeducativa (SA.5).

(32) Psicología de la Educación (SA).

(33) Psicopedagogía del desarrollo I-II (SA).

1.1. Este catálogo es incompleto, porque en algunas Universidades (p.e. SA) corre de la responsabilidad de profesores del Departamento de Teoría impartir enseñanzas que llevan por título Psicología de la Educación, Psicopatología de la Educación. Del mismo modo, como hemos dicho, hemos excluido Educación Comparada, porque no todas las Universidades la han incluido en su informe. 
Este catálogo es imperfecto porque en unas Universidades hay materias que las imparten profesores del Departamento de Teoría que en otras las imparten profesores de otros departamentos: p.e., Psicobiología de la Educación, desde Psicología (SA). En otros casos se incluyen materias sin calificativo que, con dificultad podríamos incluir en este análisis, p.e., Antropología, Sociología... (SC).

1.2. La primera apreciación ante este dato directo y primario es que la dispersión terminológica no necesariamente coexiste con dispersión en los contenidos, se da más homogeneidad en éstos últimos que en los primeros, pero manifiesta claramente imperfección en la cohesión grupal de los especialistas, falta de comunicación en cuestiones curriculares, incoherencia interuniversitaria en los planes de estudio y, sobre todo, una planificación curricular que obedece más a necesidades de rotulación diferente de "plazas" que a búsqueda de validez interna de los planes de estudio.

1.3. Desde el momento en que la denominación de Teoría de la Educación es denominación genérica de Area, denominar del mismo modo una disciplina particular introduce una ambigüedad (el todo denomina al mismo tiempo una parte), cuando se da más de un contenido disciplinar diferenciado dentro del área. Ese término de T.E. puede quedar como apelativo de una disciplina global que haga presente los temas más teóricos de la educación en curriculos no propiamente pedagógicos o de iniciación, p.e., Teoría de la Educación en Filosofía (SA). Algo parecido opinamos respecto a "Introducción..." Podría ser la denominación, para una función análoga, en ámbitos como la preparación profesional de profesores de Enseñanza Media, o Enseñanza Profesional, formación de animadores socioculturales... etc. Este fenómeno no se ha producido, p.e., en el campo de la Didáctica. Como denominación es más clara, explícita, y reconocible. Se está produciendo un fenómeno por el que se concentra cada vez más la identidad de la Pedagogía en la Didáctica, según otros profesionales (especialmente psicólogos), con lo que se absorben los contenidos de la T.E. en otras áreas. Idéntica reflexión haríamos con la disciplina de Pedagogía General.

1.4. La denominación de las áreas y la adscripción de profesores y áreas a Departamentos tiene el inconveniente que la solicitud de uno, atraída por la denominación de una disciplina (v.g. Antropología, Biología de la educación...), introduce programas (tener en cuenta la libertad de cátedra) que pueden tener poco que ver con utilidades formativas y con perspectivas profesionales de los pedagogos. Por otro lado una denominación con referencia directa a un campo más general -Metodología, Antropología, Sociología, Psicología...- por mantener la perspectiva que introducen las "CC.EE.", posibilitan una reclamación razonada de profesores de otras áreas, cuya descripción en el Decreto de Areas incluye explícitamente tales denominaciones- produce un vaciamiento del contenido curricular atribuible a especialistas en T.E. Por lo que creemos que las futuras denominaciones de materias curriculares no deben construirse con esos sustantivos, sino describir, con la sobriedad de un título, la intención y objetivo expreso de la propuesta disciplinar: (Pedagogía de la Motivación en los procesos educacionales -SC.1, Pedagogía de la conducta moral-B.6... Pedagogía de los procesos cognitivos-plaza de titular en (SA).

1.5. Se dan denominaciones que obedecen más a un replanteamiento de las cuestiones teóricas de la educación desde un nuevo paradigma o teoría científica, que a la deli- 
mitación de un campo de problemas claramente diferenciable. La Pedagogía Cibernética obliga al teórico de la educación a introducir la perspectiva en sus análisis más que a crear una nueva disciplina coexistente (ocurre en B). Se han ido manteniendo en unos y otros lugares las denominaciones disciplinares más tradicionales junto a las que procedían de puntos de vista más actuales. Desde el momento en que el adulto (denominación(10)) se incorpora a las reflexiones educativas, lo mismo decimos del anciano, debe reestructurar los contenidos de la disciplina que estudie el sujeto educativo desde un punto de vista general. Tal incorporación es parecida a la que habría que hacer con la denominación 30 de SC.5.

\section{Configuraciones disciplinares}

Considerando los planes de estudio de las disciplinas programadas, nos parece advertir varios modos de configurar internamente el campo disciplinar de la T.E. en los diferentes Centros académicos; en ocasiones, se dan varias configuraciones al mismo tiempo.

2.1. Configuración propedéutica o sistemática. Asimilamos la una a la otra, porque la estructura aparece objetivamente similar. Tanto si la disciplina se diseña como introductoria, o recapitulativa, tiene la estructura de una recopilación de los temas más prominentes que se encuentran en un recorrido por toda la geografía de los problemas pedagógicos y de la enciclopedia del conocimiento pedagógico ${ }^{2}$. (Pedagogía General, P. Sistemática, Introducción...).

2.2. Configuración de disciplina autónoma. Aparece el diseño construido desde su propia autonomía y sin tener en cuenta el resto del plan formativo. Contiene todos los elementos que definen el campo de estudio como cuerpo de conocimientos en el que trabaja un gremio científico según el criterio de la sociología de las profesiones. Aspectos epistemológicos, métodos de investigación, diferentes perspectivas desde las que estudian...(Sociología de la Educación, Antropología pedagógica).

2.3. Configuración modular. Denominamos así al señalamiento de un campo disciplinar que responde a una propuesta de conocimientos útiles, pedagógicamente significativos pero que no aspiran a ser considerados, ni ciencia, ni asignatura en un sentido tradicional, tan sólo aparecen como "unidad curricular" coherente. Un ejemplo podría ser "Sistemas de educación no formal", cuando coexiste con el resto de materias en B (Ped. social, Educación... de adultos, Ped. de animación sociocultural...).

Estos tres tipos de concebir materias del plan de estudios, aunque lógicamente parecen complementarios, en la práctica curricular son entre sí incompatibles, crean fuertes recubrimientos en la zona epistemológica y en las zonas más sistemáticas.

2. 0-1. Se organiza en Sectores Temáticos: El fenómeno educativo..., Bases biológica y psicológicas... Bases sociológicas... Fundamentos de carácter instrumental (organizativa, didáctica, económica, administrativa, legislativa). 


\section{Los temas clave}

A partir de las denominaciones de las materias programadas y de las programaciones, y hechas todas las reflexiones anteriores, se adivinan varios núcleos de trabajo investigador y académico de interés pedagógico actual y que están siendo tratados y estudiados en unos u otros lugares o en varios simultáneamente. Los presentamos por un cierto orden de generalidad:

N.O. Epistemología pedagógica. (i) Presentación de la evolución histórica de la reflexión en temas educacionales y sus modos o tipos de racionalidad, (ii) planteamientos actuales sobre la Ciencia de la Educación, la Tecnología de la Educación, la praxis reflexiva y las teorías personales o el pensamiento pedagógico de los educadores; (iii) la intencionalidad educativa: valores de la cultura, personalidad saludable, integración social...

N.1. Procesos educacionales primarios: (i) Recoge la información que contienen las descripciones de los mismos en las historias de las culturas en el pasado; (ii) las informaciones sobre procesos educativos en culturas primitivas contemporáneas; (iii) diferencias en procesos educativos en comunidades humanas culturalmente diferentes; (iv) procesos interculturales...

N.2. Relaciones mente-cuerpo: (i) Qué propiedades de la estructura que soporta el comportamiento explica el comportamiento inteligente; (ii) aspectos psicofisiológicos que explican la deficiencia mental; (iii) parámetros fundamentales de la etología animal y humana; (iv) herencia y conducta; (v) maduración, evolución... en los procesos comportamentales, del nacimiento a la muerte; (vi) educación corporal.

N.3. Pedagogía de los procesos cognitivos: (i) procesos cognitivos en el aprendizaje; (ii) estilos y diferencias cognitivas; (iii) estrategias pedagógicas de aprendizaje; (iv) tipologías de las tecnologías y estrategias de la enseñanza.

N.4. Pedagogía del comportamiento afectivo: (i) procesos afectivos en la interacción educativa educador-educando; (ii) maduración afectiva y fragilidad emocional; (iii) procesos pedagógicos de la maduración y estabilidad emocional...

N.5. Pedagogía del comportamiento moral: (i) procesos de socialización y procesos de desadaptación social; (ii) normatividad del comportamiento social; (iii) procesos del comportamiento moral; (iv) maduración y pedagogía del comportamiento moral.

N.6. La educación y su entorno: (i) influencias socioambientales en el comportamiento; (ii) intervenciones pedagógicas sobre los ambientes culturales; (iii) políticas culturales; (iv) la educación ambiental; (v) animación sociocultural; (vi) estructura social, sistemas educativos.

(Estimamos que el N.7 que con toda claridad se deduce, aquél que tiene como objeto de estudio al propio sistema de enseñanza en su estructura y planificación, quedaría más lógicamente conformado dentro de la actividad de quienes estudian disciplinas como la denominada Educación Comparada. Lo que no cabe la menor duda es que las políticas de enseñanza deben ser conocidas por los estudiantes. Hoy, los sistemas de enseñanza producen información y poseen complejidad como para que de su conocimiento y comparación con otros puede constituir una unidad disciplinar). 
N.8. Pedagogía de la comunicación: (i) Educación y comunicación oral; (ii) Educación y comunicación escrita; (iii) comunicación corporal; (iv) otras formas de comunicación; procesos y problemas básicos de la comunicación educativa.

\section{Problemas generales de los diseños}

Cuando empleamos el término problema lo hacemos en el mismo sentido en el que en el lenguaje coloquial sentenciamos "tiene o tengo un problema". Aquí es diagnóstico de mal funcionamiento por los síntomas que a continuación se identifican.

4.1. Delimitación disciplinar difusa. El diseño de Pedagogía general en 0.1 tanto se construye bajo el criterio de aspectos descriptivos del fenómeno educacional, como bajo el criterio de introducir en conceptos básicos de diferentes campos del conocimiento pedagógico. De ahí se derivan dos tipos de organización de los conceptos: aspectos del fenómeno, aspectos disciplinares diferentes. De ahí que un elemento del fenómeno, dentro de la misma disciplina, v.g. la noción de agente o foco de influencia aparecerá en la descripción del fenómeno educativo, en los aspectos sociales, en los aspectos institucionales ${ }^{3}$.... Los límites de una disciplina se construyen de manera que la responsabilidad de los diferentes profesores cubra con sus programas un campo de extensión razonable y amplia de la problemática teórica de la educación. Pero sin que se dé un punto de vista previsible en los contenidos, desde fuera. En M.6-M.7-M.8 se da el caso de una misma disciplina, en el mismo centro, con profesores diferentes, presenta diferentes planteamientos de objetivos, contenidos, diseño, amplitud y amplias zonas de no coincidencia. M.9 que es un programa de Pedagogía Social tiene más temas coincidentes con M.2. (Programa de Pedagogía General) que con M.6-M.7-M.8 (todos ellos programas de Pedagogía Social).

4.2. Recubrimientos y repeticiones originadas desde la simultánea aplicación de criterios de diseño curricular contradictorios: el propedéutico y sistemático junto al de disciplina autónoma; por ésto los problemas epistemológicos aparecen por exigencias diferentes: las de introducir y las de presentar lógicamente una disciplina. Los recubrimientos se dan dentro de un mismo centro académico. $(0.1 \text { y } 0.2)^{4}$.

4.3. Más relevancia a los intereses intelectuales de los profesores que al equilibrio de los programas. Se evidencia que los criterios Departamentales y de Equipos docentes que se explican en Didáctica no se aplican a la enseñanza de la T.E. Es la consecuencia de la identificación gremial de los campos del diseño y la necesidad de verlos reflejados en el diseño formativo. 21 denominaciones disciplinares son propias o específicas de cada Centro académico.

4.4. Aparece falta de comunicación entre profesores como consecuencia de que no existen campos comunes de diálogo, como consecuencia de la falta de ámbitos de semejanza en los diseños; a su vez, esta carencia lleva a la falta de comunicación. Se observa una clara dependencia entre los diseños y la bibliografía de uso de los profesores; lo cual

3. 0.1 T.3 (Bipolaridad del proceso. Agentes de la educación... Los principales agentes...) T.17 (Las comunidades sociales fuente de solicitaciones, estímulos, medios...) T.20 (análisis del papel del pedagogo).

4. 0.1. y 0.2 repiten los temas en los que la sociedad es agente de influencia, y los temas epistemológicos, temas económicos, temas institucionales -familia, escuela..., temas políticos... 
no sólo se traduce en una concepción creativa de la materia, lo que sería un enriquecimiento, sino que también afecta a lo que cada quien cree que debe estudiar y enseñar, lo que lleva a la no convertibilidad de unos programas que llevan la misma denominación, pero que se desarrollan en centros diferentes. Una misma denominación disciplinar hace imprevisible los contenidos que poseen programados en distintas Universidades ${ }^{5}$. Análogamente un campo relativamente coherente de problemas puede disociarse en disciplinas diferentes, p.e., B separa Educación permanente y de Adultos, de Pedagogía Social, de Pedagogía de la Animación sociocultural, de Sistemas de Educación no formal. Ello obliga a que, de hecho, el programa se concentre en la educación compensatoria y en la formación ocupacional. Finalmente vacía de contenido la programación de la Pedagogía Social o lo lleva a numerosos recubrimientos. Un mismo capítulo, con idéntica titulación -"Fundamentos antropológicos-fundamentación antropológica"- en M.2 y M.5 aparecen en Pedagogía General y en Filosofía de la Educación, aunque ciertamente contienen epígrafes diferentes; se repite la situación en "Teleología pedagógica-... fines de la educación".

4.5. Falta de unidad sistemática en el análisis de una misma cuestión en Centros diferentes y hasta dentro de programas distintos dentro del mismo Centro ${ }^{6}$. Cualquier intento de encontrar un criterio que permitiera justificar las diferentes organizaciones curriculares en los Centros resulta imposible ${ }^{7}$. Lo que razonablemente podría plantearse como un curso de Pedagogía a matemáticos, se traducen en G.5 por la disciplina "supuestos de la educación", en el que se describen 8 perspectivas en la interpretación de la educación.

P.e., la descripción del sistema educativo español y sus aspectos comparativos se estudian en SC en Sociología de la Educación II, aunque existe una asignatura de Planificación educativa; los aspectos de la socialización se presentan en Sociología de la Educación y no en Pedagogía Social. En G.4 el sistema educativo español se estudia en una materia cuyo rótulo es Política y Legislación escolar; la mayor parte del programa podría ser considerado como de teorías de la educación en perspectiva sociopolítica ${ }^{8}$.

5. Lo que G.1 entiende por contenido de una P. Sistemática, o el contenido de G.2 para la Introducción...(evolución del concepto de educación en la Historia, epistemología del concepto de ciencia y tecnología, y paradigmas metodológicos en la investigación educativa).

6. Un mismo problema, v.g. el concepto de educación, aparece en G.2 (Introducción...) ocupando 6 temas del programa en perspectiva histórica; sólo el epígrafe 2 de la unidad didáctica 1 en G.3 (Filosofía...). En ambos programas se repite la alusión a aspectos históricos, con requerimientos diferentes (G.3 tiene una unidad didáctica pero alude a 9 del libro de texto base). El tema "el medio sociocultural de la educación" en G.3 es el título de la unidad III de Filosofía, pero es el capítulo más específico de la Sociología... (materia que no existe en este centro).

7. P.e., SC tiene programas de Pedagogía general, Teoría de la Educación, Educación y Motivación, Educación y desenvolvimiento personal; nadie sospecharia que debajo de los rótulos de Educación y motivación y Educación y desenvolvimiento personal se esconde una psicología evolutiva y una psicología del aprendizaje y la motivación.

8. G.4 demuestra un planteamiento muy habitual en los programas analizados, las denominaciones disciplinares en cuyo rótulo intervienen sustantivos que denominan campos o ramas científicas autónomas (política, sociología... etc) hacen un largo recorrido descriptivo de ese campo de conocimiento antes de aterrizar en cuestiones educacionales (desde la epistemología de ese conocimiento, sus conceptos generales, para finalmente incidir sobre el sistema español. B.2 en la Biología de la Educación ha de recorrer, la estructura básica de los seres vivos, la célula y las funciones celulares, el sistema nervioso, el endocrino, la etología...etc. 
Este planteamiento de la Política educativa está contenido en M.3.y M.5 dentro de una materia denominada Etica y Política, la cual incluye en M.5 la educación moral y los aspectos de deontología profesional.

4.6. Es muy fácil rastrear en los diferentes programas las Secciones de Ciencias de la Educación que conviven con otras Facultades de Ciencias Humanas y Sociales, suelen aumentar las materias básicas de los diferentes currículos, p.e., SC contiene Antropología, Sociología, Psicología de la Educación, Fundamentos Biológicos..., Motivación y Educación (Psicología del aprendizaje), Educación y desenvolvimiento personal (Psicología evolutiva); algo análogo ocurre en SA. También te encuentras que una antigua cátedra, con una denominación que, en su día, era perfectamente comprensible y cumplía una función curricular explícita, se mantiene hoy y su contenido se torna ambiguo, P.e., Fundamentos de Metodología, M.1, contiene cuestiones de epistemología general, epistemología científica y epistemología pedagógica (a su vez, según el profesor será más epistemológico o más introductor a la actividad de quien estudia e investiga en la práctica pedagógica universitaria).

\section{Sugerencias de planificación didáctica}

(i) Romper los conceptos tradicionales de disciplina, locales o históricos, y elaborar las enseñanzas en torno a problemas, con la correspondiente indicación de créditos.

(ii) Consensuar grupalmente las denominaciones básicas para los ámbitos de problemas más generales y menos discutibles; al tiempo que se acuerdan los principales bloques de temas de estudio que merecen mayor acuerdo sobre su pertinencia y necesidad; evitar al identificarlos emplear circunloquios que los oculten (desenvolvimiento humano, en vez de psicología evolutiva).

(iii) Considerar que la elaboración de textos en común, o el alimento de publicaciones periódicas son un elemento básico para la configuración de este campo de conocimiento.

(iv) Plantear como proyecto común la innovación pedagógica mediante la cual el objetivo proceso de enseñanza-aprendizaje que se siga en las aulas manifieste el contenido mismo y la orientación de las enseñanzas pedagógicas que se pretenden transmitir.

(En todos los programas se indican con claridad los objetivos, programa, actividades y modo de evaluación. Pero se mantienen los indicios de dificultad que hemos creído encontrar en los programas, al confrontarlos unos con otros).

(v) Las reuniones científicas deben manifestar el estado actual de las investigaciones que los miembros del grupo o los equipos que formen estén realizando. Los trabajos sugeridos tienen el inconveniente de la improvisación o la reiteración. Serían mejor aprovechables para nuestras publicaciones periódicas. 